\title{
Antimicrobial Drug Resistance: Are we Fighting a Losing Battle?
}

\section{Erik de Leeuw*}

Institute of Human Virology \& Department of Biochemistry and Molecular Biology of the University of Maryland Baltimore School of Medicine 725 West Lombard street Baltimore, Maryland, 21201, USA

An ongoing explosion of resistant infections continues to plague global health care. Patients with weakened immunity because of chemotherapy, AIDS or organ transplantation or patients undergoing acute care in hospitals are significantly and increasingly at risk for acquiring opportunistic bacterial infections. In the USA alone, the Centers for Disease Control estimates that $\sim 1.7$ million infections per annum occur following hospitalization, with an estimated 99,000 deaths associated. In European healthcare systems, around 170,000 people acquire an infection with methicillin-resistant Staphylococcus aureus (MRSA) with $\sim 5000$ fatalities associated. In the vast majority of these cases, patients enter a healthcare facility in the hope of cure; however contract a barely treatable and potentially deadly infection, often during unrelated medical procedures. Seven leading groups of pathogens account for the increased risk for such infections, including four Gram-positive bacteria: Staphylococcus aureus, Enterococcus faecium, streptococci and coagulase-negative staphylococci and Gram-negative Enterobacteriaceae and Bacilli species. Resistance against commonly used classical antibiotics has emerged in all of these pathogens. In fact, there is no antibiotic currently in clinical use against which resistance has not been reported. Presently, the majority of the public remains unaware of this critical situation. In particular, rapidly increasing urbanization in developing nations are sites of major concern. Often, people in these locations have no access to adequate sanitation or can't afford access to health care. Additionally, the widespread practice by physicians to prescribe antibiotics in cases of viral infections puts selective pressure on antibiotics that still remain effective and it will only be a matter of time before resistance develops on a large scale.

To make matters worse, an alarming decline has occurred in the research and development of novel antibiotics, resulting in a rapid decline of the pipeline for novel antibiotics. In the United States, the Federal Drug Administration, a government agency which approves drugs for clinical use, has approved only ten antibiotics since 1998. Out of these ten drugs, only three can be classified as novel, with the remaining seven compounds being small chemical modifications of already existing antibiotics. This decline is both a result of insufficient government funding as well as the lack of investment by most major pharmaceutical companies in antibiotic research. Many pharmaceutical companies have halted the development of antibiotics altogether in favor of developing drugs against chronic disorders.

But all is not lost. While government funding is likely to further decrease in the current economic climate, small biotech companies are starting to fill the void in antibiotic research. These small companies do not differ in their approach from research programs of big pharmaceutical companies, in the sense that they too go after the three classical mechanisms for antibiotic development: bacterial protein synthesis, DNA synthesis and supercoiling and bacterial membrane biosynthesis. These smaller companies are often much more focused on specific product development and much more adaptable in their research strategy compared to larger organizations. In doing so, they are better able to manage the risk that resistance during product development occurs, a second major reason why antibiotic development has become unattractive to large pharmaceutical drug developers.

An alternative approach to antibiotics is the development and clinical use of bacteriophages as therapeutic agents. Bacteriophages specifically infect and kill bacteria, acting very similar to the way viruses attack human cells. With the exception of the former Soviet Republic of Georgia, bacteriophage therapy is not widely practiced; however this approach is gaining increasing interest. In addition to the ability to target bacterial species very specifically, this technique also makes possible the transfer of genetic material into the bacterium, a potentially powerful technique to disarm pathogens from within. Finally, continued efforts in developing effective vaccines against Gram-positive and -negative harmful bacteria are essential for our battle against the bugs.

We should realize that we are in the midst of an emerging crisis of antibiotic resistance and if we cannot come up with novel antibiotic strategies this could mean a literal return to the pre- antibiotic era for many types of infections.
*Corresponding author: Erik de Leeuw, Institute of Human Virology \& Department of Biochemistry and Molecular Biology of the University of Maryland Baltimore Schoo of Medicine 725 West Lombard street Baltimore, Maryland, 21201, USA, E-mail: edeleeuw@som.umaryland.edu

Received July 25, 2012; Accepted July 25, 2012; Published July 28, 2012

Citation: de Leeuw E (2012) Antimicrobial Drug Resistance: Are we Fighting a Losing Battle? J Bioanal Biomed S6: e001. doi:10.4172/1948-593X.S6-e001

Copyright: (C) 2012 de Leeuw E. This is an open-access article distributed under the terms of the Creative Commons Attribution License, which permits unrestricted use, distribution, and reproduction in any medium, provided the original author and source are credited. 